\title{
STATISTICS OF BINARY STARS: SEMI-MAJOR AXES
}

\author{
V. T R I M B L E \\ Dept. of Physics, University of California, Irvine, Calif. U.S.A. and Astronomy Program, \\ University of Maryland, College Park, Md, U.S.A. \\ and \\ C. CHEUNG \\ Astronomy Program, University of Maryland, College Park, Md., U.S.A.
}

\begin{abstract}
We have examined the distribution of the semi-major axes of the binary systems in the Sixth Catalogue of the Orbital Elements of Spectroscopic Binary Systems (and its extensions) and the correlation of semi-major axis with other properties of the systems. The total distribution has a single peak near $a \sin i=10^{7} \mathrm{~km}$. Evolved systems have wider separations and smaller mass ratios than unevolved systems. Among each type separately, the distribution of mass ratios is bimodal and small mass ratio is correlated with large separation. These data appear to show evidence of two mechanisms of binary system formation and of the process of mass transfer in close binaries.
\end{abstract}

\section{Introduction and History}

Paper I (Trimble, 1974) discussed the distribution of the mass ratios of the binary systems in the Sixth Catalogue of the Orbital Elements of Spectroscopic Binary Systems (Batten, 1967) and the supplements to it (Pedoussant and Ginestet, 1971; Pedoussant and Carquillat, 1973). The distribution turned out to be bimodal, the peaks occurring near $M_{2} / M_{1}=0.3$ and 1.0 . The bimodality does not appear to be an observational selection effect, and it is preserved when apparently evolved and unevolved binary systems are considered separately. This may imply that binaries are formed in two rather different ways, one producing approximately equal components, and one producing quite unequal components.

In the present paper, we investigate the distribution of the semi-major axes $(a \sin i)$ of the systems in the same data sample and compare it to the corresponding distributions for the smaller but more complete samples given by Blaauw and van Albada $(1967,1973)$ for $O$ and $B$ stars in associations and by Abt and Levy (1975a, b) for F and G main sequence stars near the Sun. Since Blaauw and van Albada have also suggested that binary stars might be formed in two ways on the basis of the bimodal distribution of semimajor axes in their sample, we also examine the correlations of semi-major axis with mass ratio.

The subject of the statistics of binary systems and their interpretation is an ancient and honorable one. Kuiper (1935), who cites still earlier work, collected data on several hundred spectroscopic and visual binaries in the solar neighborhood and attempted to allow for the incompleteness of his sample as a function of spectral type (covering A-M main sequence stars and $F$ and $G$ giants). His plot of frequency per unit interval in $\log$ $a$ vs. $\log a$ was a gaussian, peaking at $a=20 \mathrm{AU}$ and having a full width at half maximum of 3.0 in $\log a$. The chief difficulty in arriving at this distribution was that all the spectroscopic systems were on one wing of the gaussian and most of the visual systems on the other side. Thus, the shape of the curve was heavily dependent on the form of the dotted line drawn between the two classes of objects. 
Kuiper concluded, partly on the basis of this unified distribution for separations of both visual and spectroscopic binaries, that the binary stars were a single class of objects having common origins. Beer (1956) similarly suggested that, since the mass distribution properties of binaries did not depend on their separations or periods in the data available to him, they are, therefore, a single group.

Two major efforts have been made to bridge the gap between visual and spectroscopic binaries. Blaauw and van Albada $(1967,1973)$ examined the $O$ and early B stars in five associations and believe they have identified virtually all the binaries with $M_{2} / M_{1} \geqslant 0.2$ and separations less than $0.2-0.3 \mathrm{AU}$. They find that the frequency of spectroscopic binaries is declining much too fast with increasing separation (where their data are still reasonably complete) to be connected up smoothly with the frequency of visual binaries in the same associations (for which they claim only a lower limit). They, therefore, conclude that the distribution of semi-major axes is bimodal. They also find that, among the spectroscopic systems, the distribution in mass among the secondaries is not like that for single stars or for binary primaries. There are far fewer low-mass stars among the secondaries than one would expect from, eg, the Initial Mass Function given by Salpeter (1955).

Abt and Levy (1975) have examined the 135 F3-G2 IV and V stars brighter than $V=5.5$ and north of declination $-20^{\circ}$, identified 88 spectroscopic and visual binaries and common proper motion pairs within that group, and attempted to allow for incompleteness as a function of mass ratio and semi-major axis. Their plot of the observed number of systems versus period shows a single peak at $P=14 \mathrm{yr}$, a slight decline toward shorter periods, and a steeper decline towards longer periods (the number dropping to zero at $P=3 \times 10^{7} \mathrm{yr}$ ). At first glance, this appears consistent with the conclusions of Kuiper (1935) and Beer (1956) and inconsistent with those of Blaauw and van Albada (1967, 1973), except, of course, that OB stars and FG stars might genuinely be different. If, however, the data of Abt and Levy are used to calculate $a \sin i$ for the systems and its distribution plotted, two or more peaks appear in the distribution. Abt and Levy also find a significant difference in mass ratios between short and long period systems. The secondaries of the long period (wide) binaries show a distribution in mass not unlike the Salpeter (1955) birth-rate function or the van Rhijn (1936) luminosity function for single stars or primaries. But the secondaries of the short period (close) systems show the deficiency of low mass stars found by Blaauw and van Albada among OB spectroscopic binaries. One of the purposes of our investigation will be to look for similar effects in the larger and more heterogeneous Catalogue sample.

\section{The Catalogue Data}

The semi-major axis (in $\mathrm{km}$ ) of a spectroscopic binary is given by

$$
a \sin i=13751 K_{1} P\left(1-e^{2}\right)^{\frac{1}{2}}(1+1 / \alpha),
$$

where $K_{1}$ is the amplitude of the velocity curve of the primary in $\mathrm{km} \mathrm{sec}^{-1}, P$ is the period in days, $e$ is the eccentricity of the orbit, $i$ is the angle between the normal to the orbit plane and the line of sight, and $\alpha$ is the mass ratio of the spectroscopic secondary to primary (usually, but not always, less than one). We computed the values of $a \sin i$ for the 827 binary systems in the Sixth Catalogue and its extensions using this expression 
and the values of $\alpha$ from Paper I. The mass ratios, $\alpha$, are, of course, directly known for double-line spectroscopic binaries. For the single-line systems, they were calculated from the mass function, $f(M)=M_{2}^{3} \sin ^{3} i /\left(M_{1}+M_{2}\right)^{2}$, using values of $M_{1}$ estimated from the spectral types of the primaries and values of $\sin ^{3} i$ equal to the observed ones for eclipsing systems and 0.679 for the others. 0.679 is the median value of $\sin ^{3} i$ in a population which is randomly oriented in space and has discovery probability proportional to the amplitude of the radial velocity curve (as discussed in Paper I).

Table I presents the distributions of $a$ sin $i$ for the total sample and for the systems grouped by spectral type. '?' designates stars for which no luminosity class was available. The bins are logarithmic and 0.33 wide in $\log (a \sin i)$. The distributions tabulated are the fractions of each spectral type falling within each bin without any corrections whatever. Data are also given for single and double-lined systems separately and for 'evolved' and 'unevolved' systems. Systems called 'evolved' contain one or two stars of spectral types I, II, III, or WR (Wolf-Rayet). Systems called 'unevolved' consist of the single-line systems of type $\mathrm{V}$ and double-line systems with both stars of type $\mathrm{V}$ or with the primary of type IV and both more massive and hotter than type V secondary. Am stars are excluded from both samples (but have a distribution much more like the 'unevolved' stars, which is relevant to some theories of how they arise). Figure 1 shows the distributions for the total sample and the 'evolved' and 'unevolved' systems graphically. Clearly, this choice of 'evolved' and 'unevolved' systems does not put all those which have undergone mass transfer into the former class and all those which have not into the latter, but it is the nearest we can come to this desirable state of affairs with the available information about the systems.

Table I shows no very strong dependence of $a \sin i$ on spectral type, except for a tendency for the peak of the distribution to come at larger separations as we go to brighter and cooler stars. These two effects are really one, since a much larger fraction of the $G$, $K$, and $M$ stars than of the $O B$ stars are above the main sequence. The effect is seen most clearly in the samples called 'evolved' and 'unevolved'. The 'evolved' systems have significantly (at least in the statistical sense) larger values of $a \sin i$ than the 'unevolved' ones. This is consistent with the samples' being, in fact, evolved and unevolved in the mass-transfer sense, since the completion of mass transfer normally more than reverses the initial mass ratio of a system and, therefore, leaves the stars further apart than they started out (Paczyński, 1971). Observational selection undoubtedly also contributes to the effect, however. Evolved stars have, on average, sharper lines than their main sequence counterparts (because their atmospheres have lower temperatures and pressures), which facilitates the discovery of binaries with large separation and small mass ratio (i.e. small velocity amplitude). The data are, at least, not inconsistent with the conventional wisdom about mass transfer. Similarly, in Paper I, it turned out that the evolved systems tended to have slightly smaller values of mass ratio than unevolved ones, as mass transfer theory would predict.

The other feature of some interest in the data of Table I and Figure 1 is the appearance of a secondary maximum in the distribution for 'unevolved' systems in Bin 10 (separation $=10^{9}$ to $10^{9.33} \mathrm{~km}$ ). It is of marginal statistical significance $(1-1.5 \sigma)$, but may represent the bimodal distribution of separations found by Blaauw and van Albada $(1967,1973)$. The same bin contains a secondary peak of the Abt and Levy (1975) data (line $A L$ in Table I). 


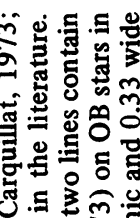

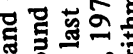

$+0$

\&

흥 잉

贾营

ठํㅇㅇㅛ

㐘

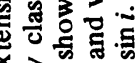

㻤。

응

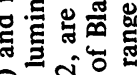

ส

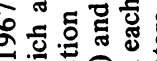

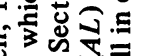
包的昰

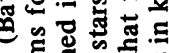

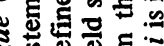

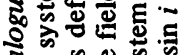

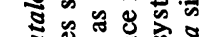

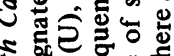

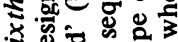

केष

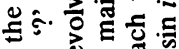

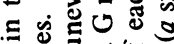

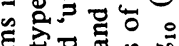

可的路

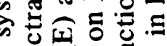

不造通

马ั

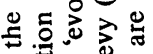

论

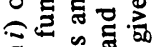

的 है

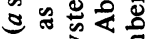

号会致声

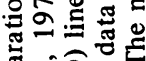

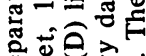

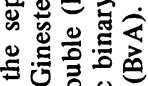

흥형

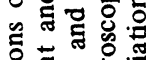

荡氞

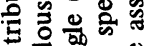

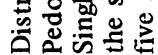

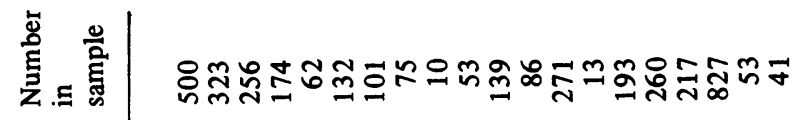

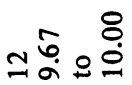

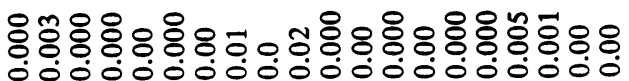

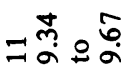

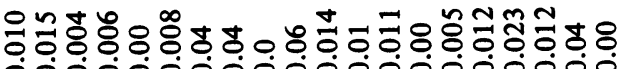

$\circ \overrightarrow{0} \sigma \stackrel{m}{m}$

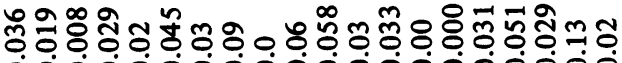
00000000000000000000

$\underset{\infty}{\infty} \stackrel{0}{\circ}$

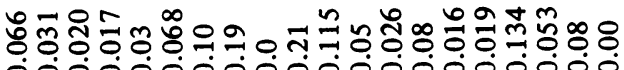

m.

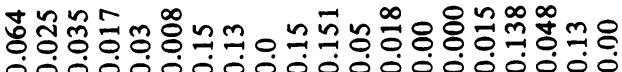

$\infty \infty$

$\overline{0} \circ \stackrel{m}{\infty}$

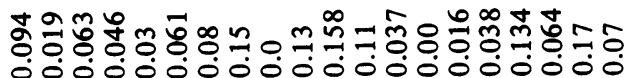

$\stackrel{5}{i}: \stackrel{8}{\infty}$

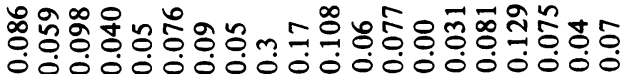

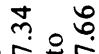

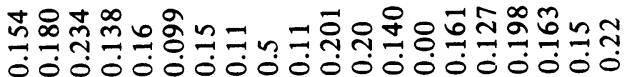

훙ํํ

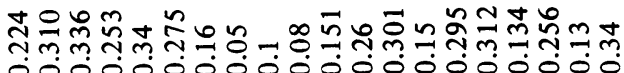

$\rightarrow+2$

కே:

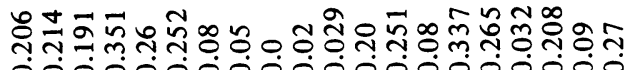

mo 8

00000000000000000000

藏。:

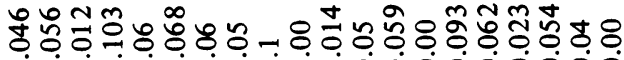
00000000000000000000

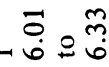

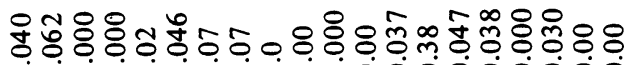
00000000000000000000

î.

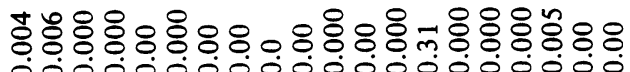

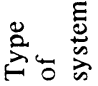

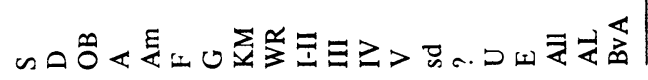




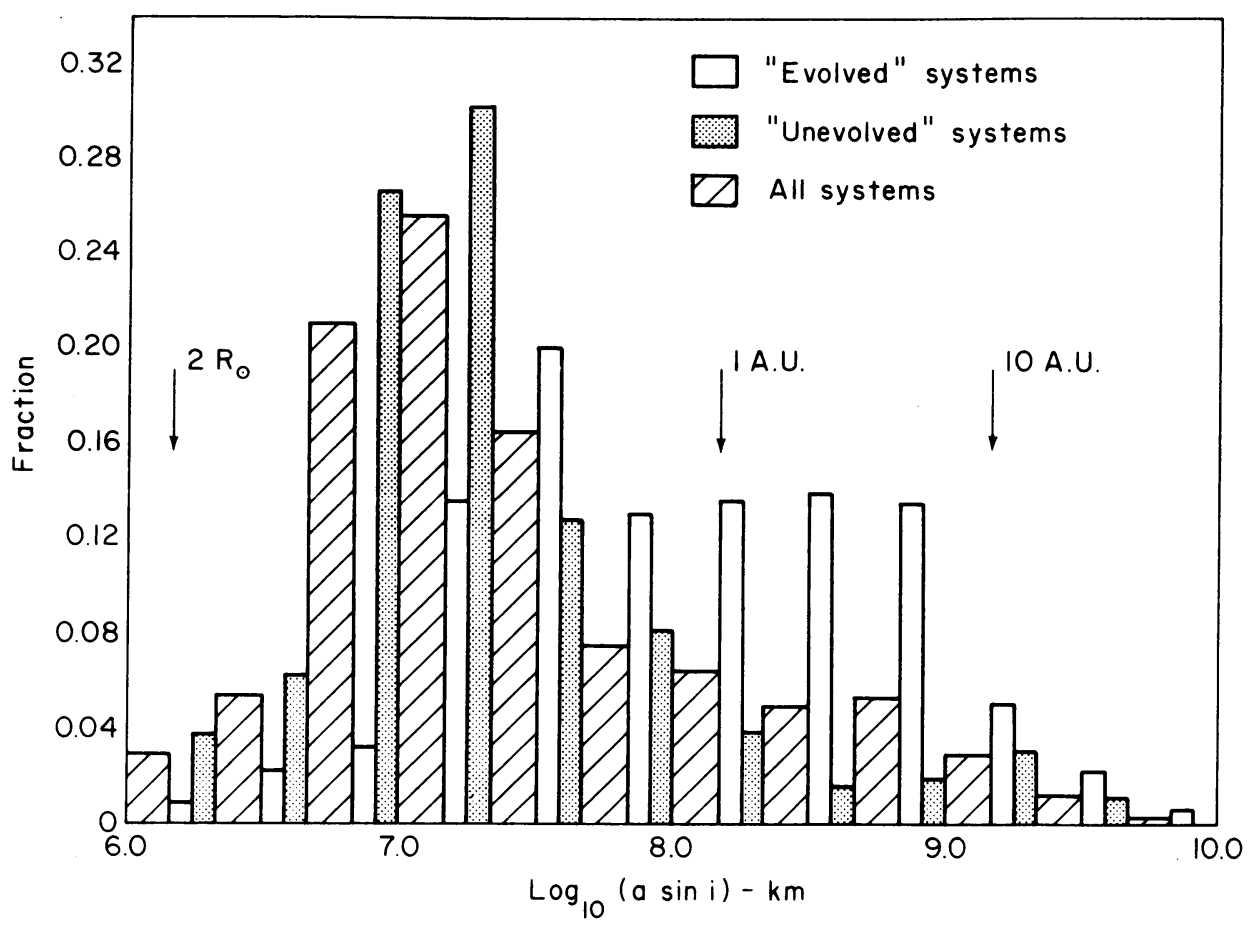

Fig. 1. The distribution of separations $(a \sin i)$ for the binary systems in the Sixth Catalogue and its extensions. The total sample, 'evolved' and 'unevolved' (as defined in Section 2) systems are plotted as lightly shaded, white, and heavily shaded bars respectively. The bins are 0.33 wide in $\log _{10}$ ( $a \sin i$ ), where $a \sin i$ is measured in kilometers. Separations corresponding to two solar radii, one, and ten astronomical units are indicated. The vertical axis is the fraction of systems in a sample falling within each bin. The most conspicuous feature is the excess of large separations among evolved systems.

Table II and Figure 2 present the data on the distribution of mass ratios as a function of separation. As in Table I and Figure 1, the numbers given are the fraction of systems of each type falling into each bin, without any corrections. The distribution of separations as a function of mass ratio can, of course, be obtained by reading down the columns of the table instead of across the lines.

There is a clear tendency visible in Table II for systems with mass ratios greater than one to have very large separations. We believe that this does not represent the real world. Abt (private communication, 1974) has pointed out that the periods given in the Catalogue for the single-line systems that appear to have mass ratios greater than one are, in many cases, much too large. A correct, smaller period would yield both a smaller separation and a smaller mass function, and, therefore, a mass ratio less than one. These systems should probably be ignored. Otherwise, there is very little correlation of mass ratio with $a \sin i$ apparent in the data. There is, however, a very strong observational selection effect against the detection of binaries with large separation and mass ratio much less than one, because such systems will appear as single-line binaries with very small velocity amplitude. Thus, the fact that the data do not show any great deficiency of such systems suggests 
V. TRIM BLE AND C. CHEUNG

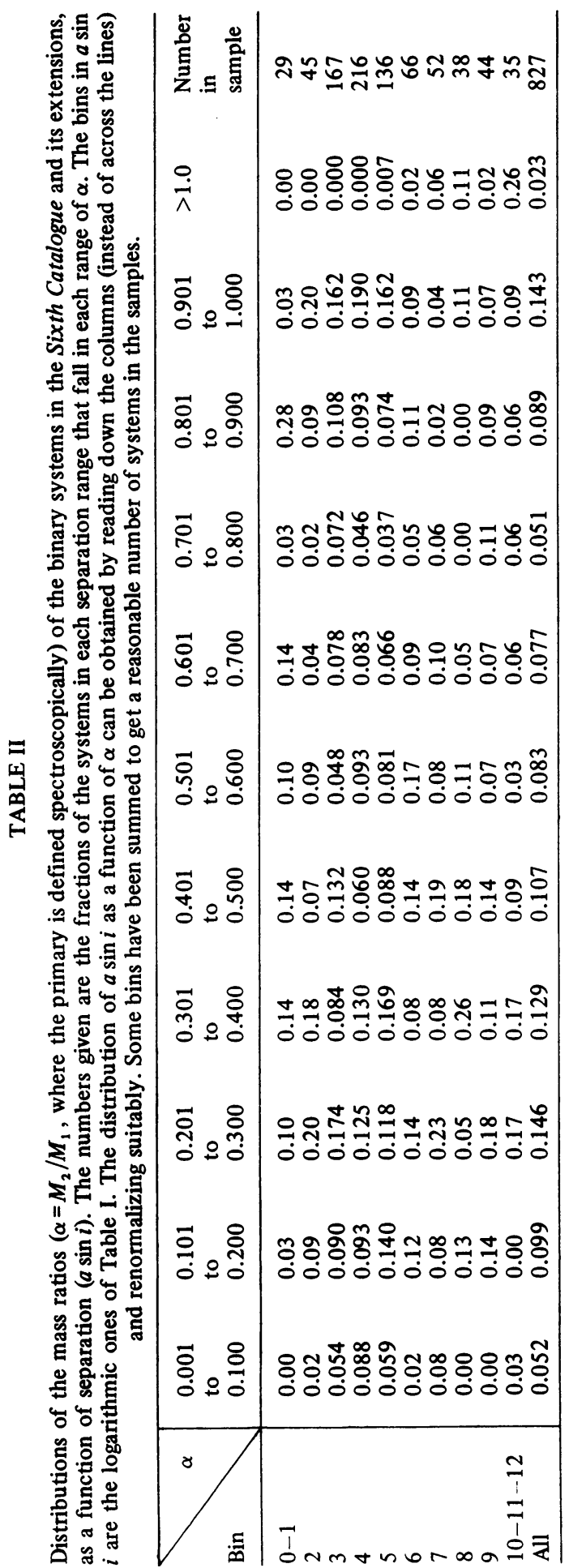




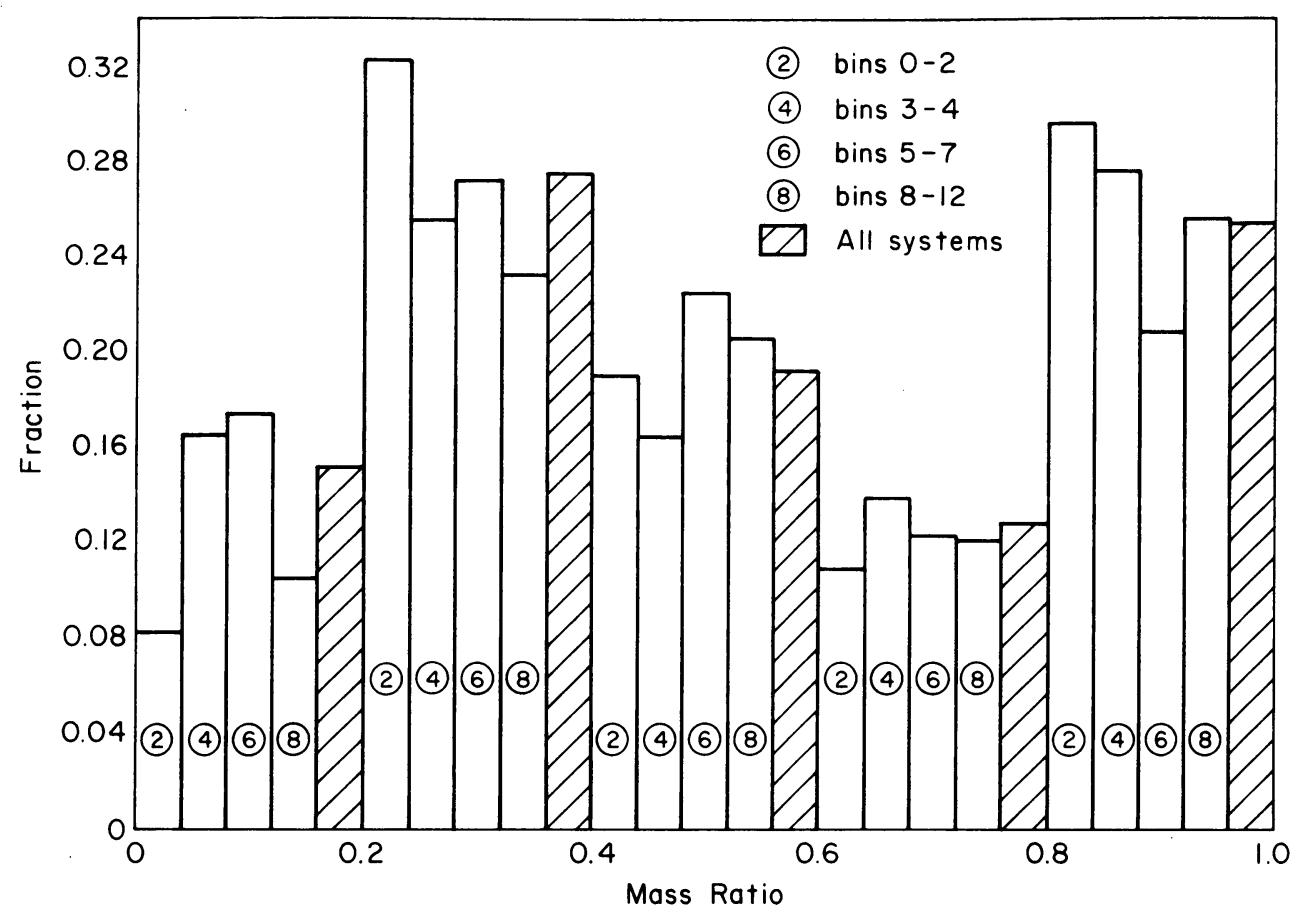

Fig. 2. The distribution of mass ratios $\left(\alpha=M_{2} / M_{1}\right)$ for the binary systems in the Sixth Catalogue and its extensions, as a function of separation $(a \sin i)$. The mass ratio ranges are 0.2 wide, and the vertical axis is the fraction of systems in each sample falling within each such range. The complete set of 827 systems is shown shaded, and the other bars represent bins $0-2,3-4,5-7$, and $8-12$ (as defined in Table I, and in order from small to large separation), containing 74, 383, 253, and 117 systems respectively. Mass ratios greater than one have been included in the $0.8-1.0$ range. All subsets show the bimodal structure of the complete set of data, and there do not seem to be any strong or systematic correlations of mass ratio with separation.

that they must be exceedingly common. This implies an anti-correlation of mass ratio with separation.

The total distribution of $a \sin i$ in the Catalogue data is quite consistent with that for the OB and FG spectroscopic binaries in the data of Blaauw and van Albada $(1967,1973)$ and Abt and Levy (1975). These are given in the last two lines of Table I. There are more wide systems among the FG stars and fewer among the OB stars than in the Catalogue sample. This is an inevitable result of the broadness of $O B$ star lines (making velocity amplitudes less than about $15 \mathrm{~km} \mathrm{~s}^{-1}$ virtually undetectable) combined with the completeness of the searches.

\section{Interpretation and Discussion}

The data presented here and in Paper I suggest (a) that evolved binary stars have wider separations and smaller mass ratios than unevolved ones, and (b) that, for the entire sample, systems with large separation tend to have smaller mass ratios than systems with small separation. The evolutionary effect is in good accord with the predictions of mass 
transfer theory, although observational selection probably also plays a part. The evolutionary effect cannot be looked for in the data either of Blaauw and van Albada or of Abt and Levy, who studied only primaries near the main sequence.

Both their sets of data do, however, show a deficiency of low-mass secondaries in close binary systems. This is evidently to be identified with our finding that small mass ratio goes with large separation. Such a correlation is predicted by mass transfer theory, a typical system having larger separation and smaller mass ratio after the mass exchange process than before.

An anti-correlation of separation and mass ratio is also predicted for unevolved binaries alone, if they are formed by the fission of a rapidly rotating fluid mass (Ostriker, private communication, 1974). This follows because the angular momentum of a binary system scales as $a^{1 / 2} \alpha /(1+\alpha)^{2}$. Thus, if a rotating gas mass with a given angular momentum fissions assymmetric fission (small mass ratio) will allow a larger separation than symmetric fission. It is, therefore, of interest to ask whether mass ratio and separation are correlated for unevolved systems alone. Abt and Levy (1975) clearly find that they are, at least for $F$ and $G$ stars, when the visual binaries are included. Their spectroscopic sample is too small to look for such an effect in it alone. The data for the 'unevolved' (defined as in Section 2) systems in the Catalogue are presented in Table III. The sample is now small enough that the statistical significance of the results is not very great, but there is a slight trend in the direction expected from observational selection; that is, for instance, the median value of $\alpha$ is lower $(0.43)$ for the closest systems and higher $(0.48)$ for the widest systems than for the sample as a whole $(0.455)$. The difference, when the data is divided in thirds, is significant at about the $1.5 \sigma$ level. This suggests that the anti-correlation of mass ratio with separation is weaker among unevolved than among evolved binaries, since it does not dominate observational selection effects in the former group. The data cannot be said to be inconsistent with the predictions of binary formation by fission, but they do not provide strong support for it in this respect either. Finally, the $\mathrm{OB}, \mathrm{FG}$, and Catalogue samples of unevolved systems all show at least weak evidence of a secondary peak in the distribution of $a \sin i$ occurring at separations of $10^{9} \mathrm{~km}$ or larger.

In Kuiper's (1935) view, the primary question was whether the binary stars formed one or two (or more) groups with one or two (or more) origins. The data from the present work, Paper I, and the more complete samples obtained by Blaauw and van Albada (1967, 1973) and Abt and Levy (1975) now seem to require us to distinguish at least three groups:

(a) Unevolved binaries formed by a process that acts rather like fission. They have relatively small separations, determined by the angular momenta of the fissioning masses, and mass ratios close to one, determined by the dynamics of the break-up. Separation and mass ratio are, perhaps weakly, anti-correlated. Most (but not necessarily all) of these systems will eventually undergo mass exchange and can, therefore, be designated Close Binary Systems.

(b) Unevolved binaries formed by a process that acts rather like separate condensation and capture in clusters. They have relatively large separations, determined by the dynamics of the cluster, and mass ratios considerably less than one, determined by the distribution of masses of the individual stars in the clusters. The predominance of small mass ratios is then a direct result of low mass stars being commoner than high mass stars, 


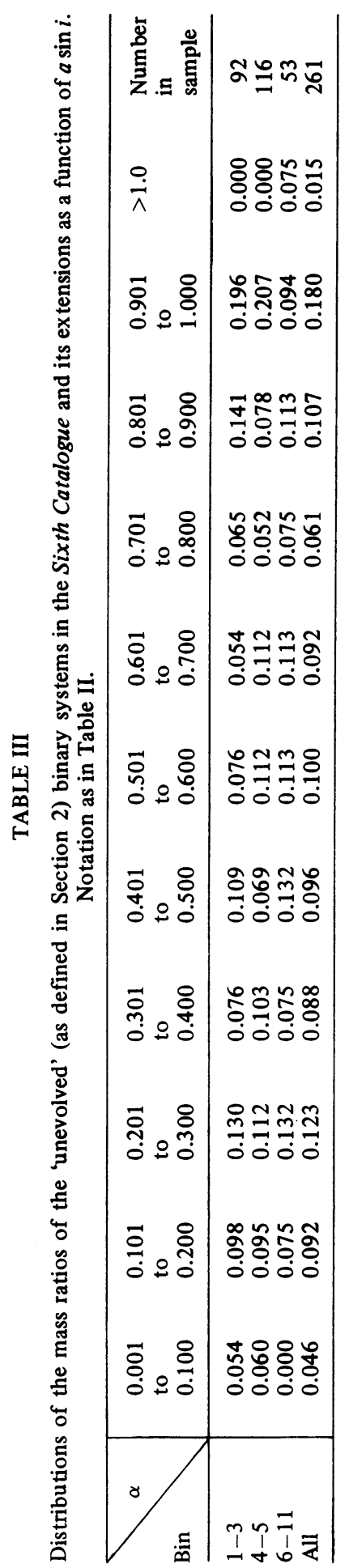


so that, if a particular future primary captures a companion at random (this appears to be what happens for all but the very few most massive stars in a cluster; Heggie, 1972, 1974), the secondary is likely to be quite a lot smaller than itself. Most of these systems will probably not undergo mass exchange.

(c) Evolved binaries, which are in the process of, or have completed, mass transfer. They have larger separations and smaller mass ratios than the systems started with. They are CBS's by definition.

A sample in which the primaries are main sequence stars later than about G2 will consist of some mix of types (a) and (b). Whether the distribution function of a given property (separation, mass ratio, period, and whatever else turns out to depend on formation mechanism) has one or two peaks will then depend on the relative proportions of the two types. Equal numbers of the two will produce a bimodal distribution, while the dominance of one or the other will yield a single peak.

For stars earlier than about $\mathrm{G} 2$, even systems chosen to have main sequence primaries will have an admixture of type (c), due to binaries in which mass transfer has been completed and the more rapidly evolving star has already achieved white dwarfhood. (Sirius is an example.). Such systems may be very common (up to 50\%) among the singleline spectroscopic binaries for some ranges of spectral type and mass ratio (Paczyński, private communication, 1974).

Spectroscopic binaries are bound to be dominated by type (a) and (c), and visual binaries by type (b), but this is not an invariable correlation (Sirius is a counter example.). Samples which include all three types may well display more than two peaks in distribution functions of various properties.

\section{Acknowledgements}

This work was begun at the Institute of Astronomy, Cambridge, England to which, and to whose Professors, D. Lynden-Bell and M. J. Rees, V.T. is, as always, grateful. Useful input on binaries and how they ought to act has been provided by Drs E. P. J. van den Heuvel, Ivan King, Jeremiah P. Ostriker, Bohdan Paczyński, Miroslav Plavec, and Daniel M. Popper.

\section{References}

Abt, H. A. and Levy, S. G.: 1975a, Bull. Amer. Astron. Soc. 7, 268.

Abt, H. A. and Levy, S. G.: 1975b, Astrophys. J. Suppl. 30, 273.

Batten, A. H.: 1967, Publ. Dom. Astrophys. Obs. 13, No. 8.

Beer, A.: 1956, Vistas Astron. 2, 1387.

Blaauw, A. and van Albada, T. S.: 1967, in A. H. Batten and J. F. Heard (eds.), The Determination of Radial Velocities and Their Application, Academic Press, New York, p. 215.

Blaauw, A. and van Albada, T. S.: 1973 (preprint).

Heggie, D. C.: 1972, Thesis, Cambridge University.

Heggie, D. C.: IAU Symp. 62, 225.

Kuiper, G. P.: 1935, Publ. Astron. Soc. Pacific 47, 38.

Paczyński, B.: 1971, Ann. Rev. Astron. Astrophys. 9, 183.

Pedoussant, A. and Carquillat, J. M.: 1973, Astron. Astrophys. Suppl. 10, 105.

Pedoussant, A. and Ginestet, N.: 1971, Astron. Astrophys. Suppl. 4, 253.

van Rhijn, P. J.: 1936, Publ. Kapteyn Astr. Lab. Groningen, No. 47.

Salpeter, E. E.: 1955, Astrophys. J. 121, 161.

Trimble, V.: 1974, Astron. J. 79, 967. 


\section{DISCUSSION}

Herczeg: This is not so much a question, rather a comment on the discovery of visually unresolved but not too close pairs of low mass ratio. The notorious difficulty of finding such stars, spectroscopically or photometrically, was mentioned in Dr Trimble's talk. It is perhaps interesting to mention that a new and apparently quite powerful way of discovering such pairs (at least in clusters) has been worked out by two of my colleagues in Norman, David Branch and Clifford Bettis. It is based on the dramatic increase of the equivalent width of the sodium D-line as we go to later spectra. A measurable excess width of this line can give away the composite character of the spectrum even for so difficult combinations as early $G+$ early $M$. A similar method can be used for discovering $A+G$ type combinations, this time based upon the $\mathrm{K}$-line. The method, which utilises narrow passband filters, will be tested at Kitt Peak Observatory this fall.

Ostriker: (1) Is there a correlation between mass and mass ratio (a) for wide systems, (b) for close binaries?

(2) The capture process will not pick out stars randomly since massive stars have a much larger cross-section for capture than low mass stars.

Trimble: (1) Not a striking one; I will try to look more carefully. (2) I used the word 'capture' carelessly. I mean some kind of separate condensation of clouds that suddenly wake up to find themselves gravitationally bound. At any rate, the data is consistent with the stars being chosen at random.

Van den Heuvel: I am somewhat puzzled by the fact that the distributions of the mass ratios of evolved as well as unevolved systems have practically the same shape. Do you have any explanation for this? Could there be 'unevolved' systems in which the invisible companion is in fact evolved, e.g. a white dwarf?

Trimble: I am a bit puzzled too. There is some difference, in the sense predicted by the Conventional Wisdom (smaller mass ratios for evolved systems) but it is not very large. Certainly the 'evolved' and 'unevolved' samples are not pure, and I quite agree that some invisible companions of main sequence stars could well be degenerate dwarfs.

Whelan: Based on your data what fraction of all binaries have $\mathrm{P}$ in range 1 yr $<P<10$ yr? These might be candidates for case $\mathrm{C}$ mass exchange.

Trimble: About 5\% of the Catalogue systems, according to Batten. This is exceedingly large, considering the strong selection effects against finding them.

Gursky: I wish to ask an historical question not directly related to your current paper. Some time ago you and Thorne presented a list of binary systems in which there was a massive unseen companion which was therefore a candidate for a black hole. Zeldovich and Guseynov did a similar piece of work. I was wondering what is the status of these lists?

Trimble: Both Abt and Hall have been re-studying many of these systems. They find, in many cases, that either the stars are not convincing spectroscopic binaries at all, or that the Catalogue periods are much too long. A corrected period yields a smaller mass function and, therefore, a smaller mass ratio. A few candidate systems remain, and I can see no way to exclude the presence of black holes in them. But I cannot exclude other explanations either. At most one of the candidates is in the error box of a $3 \mathrm{U} \mathrm{X}$-ray source.

Bolton: Is it possible that the peak you find at low mass ratios has been biased by the inclusion of an abnormal number of Algols in the spectroscopic catalogue?

Trimble: The sample includes virtually all stars with published orbital elements. This biases the sample in favour of (1) double-line and eclipsing systems, which are easy to identify as binaries (2) massive systems, whose velocity amplitude will be large (3) luminous systems, which can be studied over a large volume of space, and (4) probably other things. The peak at low mass-ratio consists primarily of single-line, non-eclipsing stars, which are, of course, the majority of all systems. This excludes Algols by definition. 